\title{
EXISTENCE OF POSITIVE SOLUTIONS FOR A CLASS OF SEMIPOSITONE PROBLEMS WITH KIRCHHOFF OPERATOR
}

\author{
Giovany M. Figueiredo, Eugenio Massa and Jefferson A. Santos \\ Universidade de Brasília, Departamento de Matemática \\ 70.910-900, Brasília - DF, Brazil; giovany@unb.br \\ Universidade de São Paulo - Campus de São Carlos, Departamento de Matemática \\ Caixa Postal 668, 13560-970, São Carlos SP, Brazil; eug.massa@gmail.com \\ Universidade Federal de Campina Grande, Unidade Acadêmica de Matemática \\ 58429-970, Campina Grande - PB, Brazil; jefferson@mat.ufcg.edu.br
}

\begin{abstract}
In this work we show a result of existence of positive solution for the following nonlocal problem of Kirchhoff type

$$
-M\left(\int_{\Omega}|\nabla u|^{2} d x\right) \Delta u=f(u)-a \text { in } \Omega, \quad u=0 \text { on } \partial \Omega,
$$

where $\Omega \subset \mathbf{R}^{N}$ is a smooth bounded domain, $M, f$ are continuous nonnegative functions and $a>0$. By using mainly variational methods, we prove the existence of a solution for $a$ small enough, under two different sets of hypotheses, which generalize the classical superlinear and sublinear problems.
\end{abstract}

\section{Introduction}

In this paper we study the existence of positive weak solutions for the semipositone problem with Kirchhoff type, possibly degenerate, nonlocal term

$$
\begin{cases}-M\left(\int_{\Omega}|\nabla u|^{2} d x\right) \Delta u=f(u)-a & \text { in } \Omega, \\ u(x)>0 & \text { in } \Omega, \\ u=0 & \text { on } \partial \Omega\end{cases}
$$

where $\Omega \subset \mathbf{R}^{N}, N \in \mathbf{N}$, is a smooth bounded domain with smooth boundary denoted by $\partial \Omega, f:[0,+\infty) \rightarrow[0,+\infty)$ is a continuous function with subcritical growth, $a>0$ and the function $M:[0,+\infty) \rightarrow[0,+\infty)$ is also continuous.

We will prove, via variational methods and with the help of some regularity theory, a-priori estimates and comparison methods, the existence of a positive regular solution for positive small values of the parameter $a$, under two different sets of hypotheses, which generalize the model problem $-\Delta u=u^{q-1}-a$, respectively, in the superlinear $(q>2)$ and the sublinear $(1<q<2)$ case.

The main feature of problem $\left(\mathrm{P}_{a}\right)$ is the presence of the term $M\left(\int_{\Omega}|\nabla u|^{2} d x\right)$, which is said to be nonlocal, since it depends not only on the point in $\Omega$ where the equation is evaluated, but on the norm of the whole solution. Such problems are usually called of Kirchhoff type, as they are generalizations of the (stationary)

https://doi.org/10.5186/aasfm.2021.4640

2020 Mathematics Subject Classification: Primary 35A15; Secondary 35J25, 35J70.

Key words: Nonlocal elliptic problems, degenerate problems, variational methods, positive solutions.

G. M. Figueiredo was partially supported by CNPq, Capes and Fap-Df - Brazil.

E. Massa was supported by: grant \#2019/19699-0, São Paulo Research Foundation (FAPESP), and grant \#303447/2017-6, CNPq/Brazil. 
Kirchhoff equation, originally proposed in [24] as an improvement of the vibrating string equation, in order to take into account the variation in the tension of the string due to the variation of its length with respect to the unstrained position.

Our results include the original nonlocal term $M(t)=c+b t$ with $c, b>0$ proposed by Kirchhoff in [24], but also admit the possibility for $M$ to be degenerate (see the model problems in Section 1.2).

Many other physical phenomena can be modeled through nonlocal equations similar to $\left(\mathrm{P}_{a}\right)$ (see examples in $[33,18]$ ), and interesting mathematical questions also arise.

For more recent literature about such Kirchhoff type problems we cite the works $[2,27,16,15,8,13,32,31,5,4,30,21,28,22]$, which deal with the existence of solutions with various types of nonlinearities $f$ and use mainly variational methods. Among them, we refer to $[15,31,4,30,28,22]$ for considering also the case where the nonlocal term $M$ is degenerate.

On the other hand, studies on positone problems are classic and very current. For example, considering the Laplacian operator, the authors in [23] show existence of radial solution in a ball or an annulus. For the case with the p-Laplacian operator, the authors in [29] found a positive solution in the critical case. A uniqueness result in exterior domains was proved in [14]. The version in Orlicz-Sobolev space was studied in [3]. Other interesting results can be seen in [1], [6], [9], [10], [11], [12], [17] and the references therein.

1.1. Statement of the results. We define the two primitives $F(t)=\int_{0}^{t} f(\tau) d \tau$ and $\mathcal{M}(t)=\int_{0}^{t} M(\tau) d \tau$ and we assume througout the paper the following two conditions:

$\left(H_{0}\right) M, f:[0,+\infty) \rightarrow[0,+\infty)$ are continuous, $M(t)>0$ for $t>0$ and $f \not \equiv 0$;

$\left(f_{s c}\right)$ there exists $q<2^{*}$ such that $\limsup _{t \rightarrow \infty} \frac{f(t)}{t^{q-1}}<\infty$,

where, for $N \geq 3,2^{*}=\frac{2 N}{N-2}$ is the critical Sobolev exponent, while for $N=1,2$ we will take $2^{*}=\infty$.

In our first setting we will also assume

$\left(M_{0}\right)$ there exists $r \in[2, q)$ such that $\liminf _{t \rightarrow 0} \frac{\mathcal{M}\left(t^{2}\right)}{t^{r}}>0$;

$\left(f_{0}\right) \quad f(0)=0$ and $\lim _{t \rightarrow 0} \frac{F(t)}{t^{r}}=0$.

Moreover, in order to obtain the required compactness condition we assume

$\left(K_{A R}\right)$ there exist $\theta>1, D, \beta, t_{0}>0$ such that

(i) $\theta F(t)-f(t) t \leq 0$ for every $x \in \Omega, t>t_{0}$,

(ii) $\frac{\theta}{2} \mathcal{M}\left(t^{2}\right)-M\left(t^{2}\right) t^{2} \geq \beta t-D \quad$ for every $t \geq 0$,

and finally, we will also impose

$\left(M_{\infty}\right)$ there exist $\theta_{1} \in(1, \theta)$ such that $\limsup _{t \rightarrow+\infty} \frac{\mathcal{M}\left(t^{2}\right)}{t^{\theta_{1}}}<\infty$. 
Remark 1. Observe that by $\left(K_{A R}\right)$ and $\left(M_{\infty}\right)$, using also that $f \not \equiv 0$, there exist $A_{1}, B_{1}>0$ such that

$$
\begin{aligned}
F(t) & \geq A_{1}|t|^{\theta}-B_{1}, \quad t \geq 0, \\
\frac{1}{2} \mathcal{M}\left(t^{2}\right) & \leq A_{1}|t|^{\theta_{1}}+B_{1}, \quad t \geq 0 .
\end{aligned}
$$

Actually, (1.2) with $\theta$ instead of $\theta_{1}$ would be a consequence of $\left(K_{A R^{-}}\right.$ii), so $\left(M_{\infty}\right)$ imposes a stronger growth condition than $\left(K_{A R}\right.$-ii). In fact, the two conditions are independent: the former is required in order to guarantee that the functional we will be working with is not bounded from below, while the latter is used in the proof of the (PS)-condition.

Condition $\left(f_{s c}\right)$ imposes a subcritical growth to $f$ and implies that $\theta<2^{*}$, while the conditions $\left(M_{0}\right)$ and $\left(f_{0}\right)$ will produce a "range of mountain" geometry around the origin for the functional, which completes the mountain pass structure.

In our second setting we still assume $\left(H_{0}\right)$ and $\left(f_{s c}\right)$, moreover we assume $\left(\widetilde{M}_{0}\right)$ there exists $r \in\left[2,2^{*}\right)$ such that $\limsup _{t \rightarrow 0} \frac{\mathcal{M}\left(t^{2}\right)}{t^{r}}<\infty$;

$$
\left(\widetilde{f}_{0}\right) \quad f(0)=0 \text { and } \lim _{t \rightarrow 0} \frac{F(t)}{t^{r}}=\infty,
$$

and in order to obtain coercivity we assume

$\left(K_{C}\right)$ there exists $\widetilde{r}>q$, where $q$ is the exponent from $\left(f_{s c}\right)$, such that

$$
\liminf _{t \rightarrow+\infty} \frac{\mathcal{M}\left(t^{2}\right)}{t^{\widetilde{r}}}>0
$$

Remark 2. In this setting, the conditions $\left(\widetilde{M}_{0}\right)$ and $\left(\widetilde{f}_{0}\right)$ will produce a situation where the origin is not a local minimum for the functional while, as observed above, $\left(K_{C}\right)$ will make the functional coercive, in view of $\left(f_{s c}\right)$. It will be then possible to obtain solutions via minimization.

Remark 3. Our techniques could be extended to work for the $p$-Laplacian operator and considering more general nonlinearities $f(x, t)$ depending also on $x \in \Omega$, however, we chose to work in the setting with the Laplacian and autonomous nonlinearity, in order to keep the presentation more clear and avoid some technicalities.

Our main results are the following.

Theorem 1.1. Assume the conditions $\left(H_{0}\right),\left(f_{s c}\right),\left(f_{0}\right),\left(M_{0}\right),\left(K_{A R}\right)$ and $\left(M_{\infty}\right)$. Then there exist $a^{*}>0$ and $\gamma \in(0,1)$ such that, if $a \in\left(0, a^{*}\right)$, problem $\left(\mathrm{P}_{a}\right)$ has a positive weak solution $u_{a} \in C^{1, \gamma}(\Omega)$.

Theorem 1.2. Assume the conditions $\left(H_{0}\right),\left(f_{s c}\right),\left(\widetilde{f}_{0}\right),\left(\widetilde{M}_{0}\right)$ and $\left(K_{C}\right)$. Then there exist $a^{*}>0$ and $\gamma \in(0,1)$ such that, if $a \in\left(0, a^{*}\right)$, problem $\left(\mathrm{P}_{a}\right)$ has a positive weak solution $u_{a} \in C^{1, \gamma}(\Omega)$.

The paper is structured as follows. First, in Section 1.2, we present some model problems that fit in the conditions of the main theorems. Then, in Section 2, we define an auxiliary problem for which we obtain a solution for suitably small values of the parameter $a>0$; in Section 3 we obtain several estimates for such solutions, and in particular we prove, in Lemma 3.2, that they are uniformly bounded in a suitable Hölder space. Finally, in Section 4, we prove the main theorems by showing that the solutions of the auxiliary problem are positive, and then they are actually solution of problem $\left(\mathrm{P}_{a}\right)$, at least for small $a$. 
Throughout the paper we will denote by $\|u\|=\left(\int_{\Omega}|\nabla u|^{2} d x\right)^{1 / 2}$ the norm in $H_{0}^{1}(\Omega)$ and by $\|u\|_{s}$ the $L^{s}$-norm. We will also use the letters $C, c$ to denote generic positive constants which may vary from line to line.

1.2. Model problems. As stated in the introduction, the local prototype for the equations we are considering is

$$
-\Delta u=u^{q-1}-a,
$$

where, respectively, $q \in\left(2,2^{*}\right)$ for Theorem 1.1 and $q \in(1,2)$ for Theorem 1.2.

More in general, consider the nonlocal problem

$$
-\left(c+b\|u\|^{\omega_{0}-2}\right) \Delta u=u^{q-1}-a
$$

with $c \geq 0, b>0$ and $\omega_{0}>2$. For $c>0$ and $\omega_{0}=4$, the left hand side is the original nonlocal term proposed by Kirchhoff in [24]. On the other hand, if $c=0$ then the nonlocal term $M$ in (1.4) is degenerate at the origin.

For (1.4) one has $\mathcal{M}\left(t^{2}\right)=c t^{2}+\frac{2 b}{\omega_{0}} t^{\omega_{0}}$, then for Theorem 1.1 one can take $2<$ $\omega_{0}<q<2^{*}$, actually hypothesis $\left(M_{0}\right)$ holds true with $r=\omega_{0}$, while $\left(K_{A R}\right)$ and $\left(M_{\infty}\right)$ hold with $\omega_{0} \leq \theta_{1}<\theta<q$. For Theorem 1.2 instead, one can take $q \in(1,2)$ if $c>0$ and $q \in\left(1, \omega_{0}\right)$ in the degenerate case $c=0$, actually hypothesis $\left(\widetilde{M}_{0}\right)$ holds true with $r=2$, but also with $r=\omega_{0}$ if $c=0$, while $\left(K_{C}\right)$ holds with $\widetilde{r}=\omega_{0}$. It is worth noting that in this last case it is possible to take a linear $f$ :

$$
-\|u\|^{\omega_{0}-2} \Delta u=u-a .
$$

A further model, where instead $M$ is degenerate at infinity, is

$$
-\frac{\Delta u}{\left(\|u\|^{2}+1\right)^{1-\omega_{\infty} / 2}}=u^{q-1}-a
$$

with $1<\omega_{\infty}<2$. In this case $\mathcal{M}\left(t^{2}\right)=\frac{2}{\omega_{\infty}}\left[\left(t^{2}+1\right)^{\omega_{\infty} / 2}-1\right]$.

For Theorem 1.1 one can take $q \in\left(2,2^{*}\right)$ : actually hypothesis $\left(M_{0}\right)$ holds true with $r=2$, while $\left(K_{A R}\right)$ and $\left(M_{\infty}\right)$ hold with $\omega_{\infty} \leq \theta_{1}<\theta<q$; on the other hand, for Theorem 1.2 hypothesis $\left(\widetilde{M}_{0}\right)$ holds true with $r=2$ and $\left(K_{C}\right)$ with $\widetilde{r}=\omega_{\infty}$, then we can take $q \in\left(1, \omega_{\infty}\right)$.

By combining the above cases one can also consider a problems degenerate at both the origin and infinity, such as

$$
-\left(\min \left\{\|u\|^{\omega_{0}-2},\|u\|^{\omega_{\infty}-2}\right\}\right) \Delta u=u^{q-1}-a
$$

with $1<\omega_{\infty}<2<\omega_{0}$ : then for Theorem 1.1, one needs $q \in\left(\omega_{0}, 2^{*}\right)$, since hypothesis $\left(M_{0}\right)$ holds true with $r=\omega_{0}$, and $\left(K_{A R}\right)$ and $\left(M_{\infty}\right)$ with $\omega_{\infty} \leq \theta_{1}<\theta<q$; for Theorem 1.2 instead one needs $1<q<\omega_{\infty}$.

As a final example, with the same left hand side as (1.5) but with nonhomogeneous functions $f$, one can consider in Theorem 1.1 an asymptotically linear nonlinearity, for instance

$$
-\frac{\Delta u}{\left(\|u\|^{2}+1\right)^{1-\omega / 2}}=\frac{u^{2}}{1+u}-a .
$$

with $1<\omega<2$, actually the hypotheses now hold with $q \in\left(2,2^{*}\right), r=2$ and $\omega \leq \theta_{1}<\theta<2$. 
In Theorem 1.2 one can also take a nonlinearity which goes to zero at infinity, for instance

$$
-\frac{\Delta u}{\left(\|u\|^{2}+1\right)^{1-\omega / 2}}=\frac{\sqrt{u}}{1+u}-a .
$$

with $1<\omega<2$, actually the hypotheses now hold with $q=1 / 2, r=2$ and $\widetilde{r}=\omega$.

\section{Preliminary results}

In the sequel, we say that $u \in H_{0}^{1}(\Omega)$ is a weak solution for $\left(\mathrm{P}_{a}\right)$ if $u$ is a continuous positive function that verifies

$$
M\left(\int_{\Omega}|\nabla u|^{2} d x\right) \int_{\Omega} \nabla u \nabla \varphi d x=\int_{\Omega}(f(u)-a) \varphi d x, \quad \varphi \in H_{0}^{1}(\Omega) .
$$

In this section, we denote by $f_{a}: \mathbf{R} \rightarrow \mathbf{R}$ the continuous functions given by

$$
f_{a}(t)= \begin{cases}f(t)-a & \text { if } t \geq 0 \\ -a(t+1) & \text { if } t \in[-1,0] \\ 0 & \text { if } t \leq-1\end{cases}
$$

$0<a<1$, and $-a=\min _{t \in \mathbf{R}} f_{a}(t)$.

Our intention is to prove the existence of a positive solution for the following auxiliary problem

$$
\begin{cases}-M\left(\int_{\Omega}|\nabla u|^{2} d x\right) \Delta u=f_{a}(u) & \text { in } \Omega \\ u(x)>0 & \text { in } \Omega \\ u=0 & \text { on } \partial \Omega\end{cases}
$$

because such a solution is also a solution of $\left(\mathrm{P}_{a}\right)$. Associated with $\left(\mathrm{AP}_{a}\right)$, we have the energy functional $I_{a}: H_{0}^{1}(\Omega) \rightarrow \mathbf{R}$ defined by

$$
I_{a}(u)=\frac{1}{2} \mathcal{M}\left(\int_{\Omega}|\nabla u|^{2} d x\right)-\int_{\Omega} F_{a}(u) d x, \quad u \in H_{0}^{1}(\Omega)
$$

where

$$
F_{a}(t)=\int_{0}^{t} f_{a}(\tau) d \tau= \begin{cases}F(t)-a t & \text { if } t \geq 0 \\ \frac{a}{2}\left(1-(t+1)^{2}\right) & \text { if } t \in[-1,0] \\ \frac{a}{2} & \text { if } t \leq-1\end{cases}
$$

As a consequence we can always estimate

$$
-a t^{+} \leq F_{a}(t) \leq \begin{cases}F(t) & \text { if } t \geq 0 \\ \frac{a}{2} & \text { if } t \leq 0\end{cases}
$$

where $t^{+}=\max \{t, 0\}$.

The functional $I_{a}$ is Fréchet differentiable with derivative $I_{a}^{\prime}$ given by

$$
\left\langle I_{a}^{\prime}(u), v\right\rangle=M\left(\int_{\Omega}|\nabla u|^{2} d x\right) \int_{\Omega} \nabla u \nabla v d x-\int_{\Omega} f_{a}(u) v d x, \quad v \in H_{0}^{1}(\Omega) .
$$

2.1. Mountain pass geometry. Throughout this section we assume the hypotheses of Theorem 1.1. The next two lemmas will be useful to prove that in this case $I_{a}$ verifies the mountain pass geometry. 
Lemma 2.1. There exist $\rho, a_{1}, \alpha>0$ such that

$$
I_{a}(u) \geq \alpha, \quad \text { for }\|u\|=\rho \text { and any } a \in\left[0, a_{1}\right) .
$$

Proof. Notice that, in view of $\left(f_{0}\right),\left(f_{s c}\right)$ and $(2.3)$, given $\epsilon>0$, there exists $C_{\epsilon}>0$ such that

$$
F_{a}(t) \leq \epsilon|t|^{r}+C_{\epsilon}|t|^{q}+a / 2, \quad \text { for all } t \in \mathbf{R} .
$$

On the other hand, $\left(M_{0}\right)$ implies that $\frac{1}{2} \mathcal{M}\left(s^{2}\right) \geq C_{1} s^{r}$ for some $C_{1}>0$ and $s$ small enough, then using also Sobolev embeddings, we get

$$
\begin{aligned}
I_{a}(u) & \geq \frac{1}{2} \mathcal{M}\left(\int_{\Omega}|\nabla u|^{2} d x\right)-\varepsilon\|u\|_{r}^{r}-C_{\epsilon}\|u\|_{q}^{q}-\frac{a}{2}|\Omega| \\
& \geq \rho^{r}\left(C_{1}-\varepsilon C-C C_{\varepsilon} \rho^{q-r}\right)-\frac{a}{2}|\Omega| .
\end{aligned}
$$

We first set $\varepsilon=C_{1} / 2 C$ and then we set $\rho$ sufficiently small such that $C C_{\varepsilon} \rho^{q-r} \leq$ $C_{1} / 4$, so that the term in parentheses is at least $C_{1} / 4$. With this, the claim is satisfied by taking $a_{1}, \alpha$ such that $\left(C_{1} / 4\right) \rho^{r}-\frac{a_{1}}{2}|\Omega|>\alpha$.

Lemma 2.2. There exists $v \in H_{0}^{1}(\Omega)$ such that $\|v\|>\rho$ and $I_{a}(v)<0$, for all $a \in\left[0, a_{1}\right)$.

Proof. Let $\varphi \in C_{0}^{\infty}(\Omega)$ be a function verifying

$$
\varphi>0 \text { in } \Omega \text { and }\|\varphi\|=1 .
$$

Note that for all $t>0$,

$$
I_{a}(t \varphi)=\frac{1}{2} \mathcal{M}\left(t^{2}\right)-\int_{\Omega} F_{a}(t \varphi) d x=\frac{1}{2} \mathcal{M}\left(t^{2}\right)-\int_{\Omega} F(t \varphi) d x+a \int_{\Omega} t \varphi d x .
$$

Estimating with (1.1) and (1.2) we get

$$
I_{a}(t \varphi) \leq A_{1} t^{\theta_{1}}+B_{1}-A_{1} t^{\theta}\|\varphi\|_{\theta}^{\theta}+t a\|\varphi\|_{1}+B_{1}|\Omega| .
$$

Since $\theta>\max \left\{\theta_{1}, 1\right\}$ and $a \in\left[0, a_{1}\right)$, we can fix $t_{1}>\rho$ large enough so that $I_{a}(v)<0$, where $v=t_{1} \varphi \in H_{0}^{1}(\Omega)$.

In the sequel, we are going to study the boundedness of (PS) sequences of $I_{a}$. To do this, observe that $\left(K_{A R^{-i}}\right)$ yields that also $f_{a}$ satisfies the famous condition due to Ambrosetti-Rabinowitz, that is, there exists $T_{a_{1}} \in \mathbf{R}$, dependeing on $a_{1}$, such that

$$
\theta F_{a}(t) \leq t f_{a}(t)+T_{a_{1}}, \quad t \in \mathbf{R} \text { and } a \in\left[0, a_{1}\right) .
$$

Lemma 2.3. The functional $I_{a}$ satisfies the Palais-Smale condition for all $a>0$.

Proof. The proof was already given in [22, Proposition 4.1]. We give it here for sake of completeness. Let $\left\{u_{n}\right\}$ be a sequence in $H_{0}^{1}(\Omega)$ such that $\left|I_{a}\left(u_{n}\right)\right| \leq \Upsilon$, for some $\Upsilon>0$ and $I_{a}^{\prime}\left(u_{n}\right) \rightarrow 0$. We then estimate $\left|\theta I_{a}\left(u_{n}\right)-I_{a}^{\prime}\left(u_{n}\right)\left[u_{n}\right]\right|$ as

$\left|\left(\frac{\theta}{2} \mathcal{M}\left(\left\|u_{n}\right\|^{2}\right)-M\left(\left\|u_{n}\right\|^{2}\right)\left\|u_{n}\right\|^{2}\right)-\int_{\Omega}\left(\theta F_{a}\left(x, u_{n}\right)-f_{a}\left(x, u_{n}\right) u_{n}\right) d x\right| \leq C+\varepsilon_{n}\left\|u_{n}\right\|$, with $\epsilon_{n} \rightarrow 0$, so that, using $\left(K_{\left.A R^{-i i}\right)}\right.$ and (2.5), we obtain

$$
\beta\left\|u_{n}\right\| \leq C^{\prime}+\varepsilon_{n}\left\|u_{n}\right\|,
$$

which implies that $\left\|u_{n}\right\|$ is bounded.

Then, up to a subsequence, we have that

$$
\left\|u_{n}\right\| \rightarrow \Upsilon \geq 0
$$


On the other hand by standard arguments, in view of the subcriticality of $f$ given in $\left(f_{s c}\right)$, it follows that, up to a subsequence, $u_{n}$ converges weakly in $H_{0}^{1}(\Omega)$ to some $u$ and from $\left|I_{a}^{\prime}\left(u_{n}\right)\left[u_{n}-u\right]\right| \rightarrow 0$ and $\left(f_{s c}\right)$, one obtains

$$
M\left(\left\|u_{n}\right\|^{2}\right) \int_{\Omega} \nabla u_{n} \nabla\left(u_{n}-u\right) d x \rightarrow 0 \quad(n \rightarrow \infty) .
$$

If $\Upsilon=0$, then from (2.7) the proof is over. If $\Upsilon>0$ by $\left(H_{0}\right)$ we obtain $u_{n} \rightarrow u$ in $H_{0}^{1}(\Omega)$ in the proof is over.

We will now obtain a solution for Problem $\left(\mathrm{AP}_{a}\right)$, by the Mountain Pass Theorem. Below we will make explicit the dependence of the constants on the bounded interval $[0, \bar{a})$ where the parameter $a$ is taken, by using as subscript its endpoint, which we still have to fix, while we will not mention their dependence on $M, \Omega$ and $f$.

Lemma 2.4. There exists a constant $C_{a_{1}}>0$ such that $\left(\mathrm{AP}_{a}\right)$ has a solution $u_{a} \in H_{0}^{1}(\Omega)$ satisfying $0<\alpha \leq I_{a}\left(u_{a}\right) \leq C_{a_{1}}$, for every $a \in\left[0, a_{1}\right)$.

Proof. The Lemmas 2.1, 2.2 and 2.3 guarantee that we can apply the Mountain Pass Theorem due to Ambrosetti-Rabinowitz [7] to show the existence of a solution $u_{a} \in H_{0}^{1}(\Omega)$ for all $a \in\left[0, a_{1}\right)$ with $I_{a}\left(u_{a}\right)=d_{a} \geq \alpha>0$, where $d_{a}$ is the mountain pass level associated with $I_{a}$.

Now, taking $\varphi \in C_{0}^{\infty}(\Omega)$ as in the proof of Lemma 2.2, $t>0$, and estimating as in $(2.4)$, we see that $I_{a}(t \varphi)$ is bounded from above, uniformly if $a \in\left[0, a_{1}\right)$. Then the mountain pass level is also estimated in the same way:

$$
0<\alpha \leq d_{a}=I_{a}\left(u_{a}\right) \leq \max \left\{I_{a}(t \varphi) ; t \geq 0\right\} \leq C_{a_{1}} .
$$

The next lemma establishes a very important estimate involving the Sobolev norm of the solution $u_{a}$ for $a \in\left[0, a_{1}\right)$.

Lemma 2.5. There exist constants $k_{a_{1}}, K_{a_{1}}$, such that $0<k_{a_{1}} \leq\left\|u_{a}\right\| \leq K_{a_{1}}$ for all $a \in\left[0, a_{1}\right)$.

Proof. By Lemma 2.4 we have

$$
\begin{aligned}
C_{a_{1}} & \geq I_{a}\left(u_{a}\right)-\frac{1}{\theta} I_{a}^{\prime}\left(u_{a}\right) u_{a} \\
& =\frac{1}{2} \mathcal{M}\left(\left\|u_{a}\right\|^{2}\right)-\frac{1}{\theta} M\left(\left\|u_{a}\right\|^{2}\right)\left\|u_{a}\right\|^{2}+\int_{\Omega}\left(\frac{1}{\theta} f_{a}\left(u_{a}\right) u_{a}-F_{a}\left(u_{a}\right)\right) d x .
\end{aligned}
$$

Then from (2.5) and $\left(K_{A R^{-}}\right.$ii) we get

$$
C_{a_{1}} \geq \frac{1}{\theta}\left(\beta\left\|u_{a}\right\|-D-T_{a_{1}}\right)
$$

and then we obtain the claimed estimate from above.

For the estimate from below, just note that by (2.3) and Sobolev embeddings

$$
\alpha \leq I_{a}\left(u_{a}\right) \leq \frac{1}{2} \mathcal{M}\left(\left\|u_{a}\right\|^{2}\right)+a \int_{\Omega} u_{a}^{+} d x \leq \frac{1}{2} \mathcal{M}\left(\left\|u_{a}\right\|^{2}\right)+C a_{1}\left\|u_{a}\right\|
$$

and the right hand side goes to zero if $\left\|u_{a}\right\|$ goes to zero.

2.2. Gobal minimum geometry. Throughout this section we assume the hypotheses of Theorem 1.2. The next two lemmas will prove that $I_{a}$ has a global minimum at a negative level.

Lemma 2.6. There exist $a_{1}, \alpha>0$ and $u_{0} \in H_{0}^{1}(\Omega)$ such that

$$
I_{a}\left(u_{0}\right) \leq-\alpha, \quad \text { for any } a \in\left[0, a_{1}\right) .
$$


Proof. Let $\varphi \in C_{0}^{\infty}(\Omega)$ be as in the proof of Lemma 2.2. As there, for $t>0$,

$$
I_{a}(t \varphi)=\frac{1}{2} \mathcal{M}\left(t^{2}\right)-\int_{\Omega} F(t \varphi) d x+a \int_{\Omega} t \varphi d x .
$$

From $\left(\widetilde{M}_{0}\right)$ and $\left(\widetilde{f}_{0}\right)$ we have that, for $t_{0}$ small enough and some constant $A>0$,

$$
\frac{1}{2} \mathcal{M}\left(t_{0}^{2}\right) \leq A t_{0}^{r} \quad \text { and } \quad \int_{\Omega} F\left(t_{0} \varphi\right) d x \geq 2 A t_{0}^{r} .
$$

Then we get

$$
I_{a}\left(t_{0} \phi\right) \leq-A t_{0}^{r}+a t_{0} \int_{\Omega} \varphi d x .
$$

Let now $\alpha=\frac{1}{2} A t_{0}^{r}>0$ and then fix $a_{1}=a_{1}\left(t_{0}\right)$ such that $a_{1} t_{0} \int_{\Omega} \varphi d x \leq \alpha$, to obtain

$$
I_{a}\left(t_{0} \phi\right) \leq-\alpha \quad \text { for } a \in\left[0, a_{1}\right) .
$$

Lemma 2.7. $I_{a}$ is coercive, uniformly with respect to $a \in\left[0, a_{1}\right)$, in fact, there exist $H, \rho>0$ independents of a such that $I_{a}(u) \geq H$ whenever $\|u\| \geq \rho$ and $a \in\left[0, a_{1}\right)$.

Proof. By $\left(f_{s c}\right),\left(K_{C}\right)$ and using $(2.3)$, we get, for suitable constants $c, C>0$,

$$
I_{a}(u) \geq \frac{c}{2}\|u\|^{\widetilde{r}}-C-C\|u\|^{q}-\frac{a}{2}|\Omega|,
$$

then the claim follows easily since $\widetilde{r}>q$.

Lemma 2.8. For every $a \in \mathbf{R}, I_{a}$ is weakly lower semicontinuos.

Proof. The proof is classical in view of $\left(H_{0}\right)$ and $\left(f_{s c}\right)$, observing that $M \geq 0$ implies that $\mathcal{M}$ is nondecreasing and then $\mathcal{M}\left(\|u\|^{2}\right) \leq \liminf \mathcal{M}\left(\left\|u_{n}\right\|^{2}\right)$ along a sequence $u_{n}$ that converges weakly to $u$.

We will now obtain a solution for Problem $\left(\mathrm{AP}_{a}\right)$ by minimization.

Lemma 2.9. There exists a constant $C_{a_{1}}>0$ such that Problem $\left(\mathrm{AP}_{a}\right)$ has a solution $u_{a} \in H_{0}^{1}(\Omega)$ satisfying $0>-\alpha \geq I_{a}\left(u_{a}\right) \geq-C_{a_{1}}$, for every $a \in\left[0, a_{1}\right)$.

Proof. The solution is obtained by minimization in view of the above lemmas. Actually the global minimum of $I_{a}$ stays below $-\alpha$ by Lemma 2.6, while the bound from below is a consequence of (2.8) with $a<a_{1}$.

We now prove that the same kind of estimate obtained in Lemma 2.5, holds true also in this case.

Lemma 2.10. There exist constants $k_{a_{1}}, K_{a_{1}}$, such that $0<k_{a_{1}} \leq\left\|u_{a}\right\| \leq K_{a_{1}}$ for all $a \in\left[0, a_{1}\right)$.

Proof. The bound from above for the norm of $u_{a}$ is a consequence of the uniform coercivity proved in Lemma 2.7, since $I_{a}\left(u_{0}\right)<0$. The bound from below follows by the same argument as in Lemma 2.5. Actually, by (2.3), Sobolev embeddings and estimating $F_{a}(t) \leq F\left(t^{+}\right)+a|t|$, we get

$$
0>-\alpha \geq I_{a}\left(u_{a}\right)=\frac{1}{2} \mathcal{M}\left(\left\|u_{a}\right\|^{2}\right)-\int_{\Omega} F_{a}\left(u_{a}\right) d x \geq-a_{1}\left\|u_{a}\right\|_{1}-\int_{\Omega} F\left(u_{a}^{+}\right) d x
$$

and again the right hand side goes to zero if $\left\|u_{a}\right\|$ goes to zero. 


\section{Further estimates for the solutions $\boldsymbol{u}_{a}$}

From now on $u_{a}$ will be the solution of Problem $\left(\mathrm{AP}_{a}\right)$ obtained either in Lemma 2.4 or in Lemma 2.9 .

As an immediate consequence of Lemma 2.5 and Lemma 2.10, in view of condition $\left(H_{0}\right)$, we obtain the following

Corollary 3.1. There exist constants $h_{a_{1}}, H_{a_{1}}$, such that $0<h_{a_{1}} \leq M\left(\left\|u_{a}\right\|^{2}\right) \leq$ $H_{a_{1}}$ for all $a \in\left[0, a_{1}\right)$.

This estimate is very important because it implies that from now on we can work as if the nonlocal term were nondegenerate and bounded, making the next steps very similar to the local case $M \equiv 1$.

Our next result ensures that $u_{a}$ belongs to $L^{\infty}(\Omega)$, and that the family $\left\{u_{a}\right\}_{a \in[0, \bar{a})}$ is a bounded set in $L^{\infty}(\Omega)$ for $\bar{a}$ small enough. This fact is crucial in our approach.

Lemma 3.2. There exists $a_{2} \in\left(0, a_{1}\right]$ and $\beta \in(0,1)$ such that $\left\{u_{a}\right\}_{a \in\left[0, a_{2}\right)} \subseteq$ $C^{1, \beta}(\Omega)$ and is a bounded set in $C^{1, \beta}(\Omega)$. In particular, there exists $C_{a_{2}}^{\infty}>0$ such that

$$
\left\|u_{a}\right\|_{\infty} \leq C_{a_{2}}^{\infty}, \quad \forall a \in\left[0, a_{2}\right) .
$$

Proof. In order to prove the lemma, it is enough to show that for any sequence $a_{j} \rightarrow 0$, the sequence of solutions $u_{j}=u_{a_{j}}$ from Lemma 2.4 (resp. Lemma 2.9) possesses a subsequence, still denoted by itself, which is bounded in $C^{1, \beta}(\Omega)$. We will do this by showing that a suitable subsequence of $u_{j}$ satisfies the conditions in [19, Proposition 3.7], which provides the claimed boundedness as a consequence of $[20,25,26]$.

As $u_{j}$ is bounded in $H_{0}^{1}(\Omega)$ by Lemma 2.5 (resp. Lemma 2.10), there is a subsequence of $\left\{u_{j}\right\}$, still denoted by itself, and $u \in H_{0}^{1}(\Omega)$ such that $u_{j} \rightarrow u$ weakly in $H_{0}^{1}(\Omega)$, strongly in $L^{\tau}(\Omega)$ for $\tau<2^{*}$ and a.e. in $\Omega$. Proceeding similar to the proof of Lemma 2.3, from $\left|I_{a_{j}}^{\prime}\left(u_{j}\right)\left[u_{j}-u\right]\right|=0$ and $\left(f_{s c}\right)$, since $f_{a_{j}}\left(u_{j}(x)\right) \rightarrow f_{0}(u(x))$ a.e. for $x \in \Omega$ one obtains

$$
M\left(\left\|u_{j}\right\|^{2}\right) \int_{\Omega} \nabla u_{j} \nabla\left(u_{j}-u\right) d x \rightarrow 0 .
$$

Note that from Corollary 3.1 that $u_{j}$ does not tend to zero and then $M\left(\left\|u_{j}\right\|^{2}\right) \rightarrow$ $c>0$ so that $\int_{\Omega} \nabla u_{j} \nabla\left(u_{j}-u\right) d x \rightarrow 0$ and then $u_{j} \rightarrow u$ strongly in $H_{0}^{1}(\Omega)$ and also in $L^{2^{*}}(\Omega)$. Hence up to further subsequence, $u_{j}^{2^{*}}$ is uniformly integrable.

Finally, from Corollary 3.1 again we get the estimate, in the weak sense,

$$
\left|-\Delta u_{a}\right|=\left|\frac{1}{M\left(\left\|u_{a}\right\|^{2}\right)} f_{a}\left(u_{a}\right)\right| \leq \frac{1}{h_{a_{1}}}\left(f\left(u_{a}\right)+|a|\right),
$$

where $f$ is subcritical by $\left(f_{s c}\right)$.

In what follows, we show an estimate from below of the norm $L^{\infty}(\Omega)$ of $u_{a}$ for $a$ small enough.

Lemma 3.3. There exists $a_{3} \in\left(0, a_{2}\right)$ and $\delta>0$ that does dependent on $a \in$ $\left[0, a_{3}\right)$, such that $\left\|u_{a}\right\|_{\infty} \geq \delta$ for all $a \in\left[0, a_{3}\right)$.

Proof. By using $u_{a}$ as a test function we have

$$
M\left(\left\|u_{a}\right\|^{2}\right) \int_{\Omega}\left|\nabla u_{a}\right|^{2} d x=\int_{\Omega} f_{a}\left(u_{a}\right) u_{a} d x
$$


By Lemma 2.5 (resp. Lemma 2.10) and Corollary 3.1 the left hand side is bounded from below by $h_{a_{1}} k_{a_{1}}^{2}$.

Let now $\delta$ be such that $f(t) t<h_{a_{1}} k_{a_{1}}^{2} /|\Omega|$ for $t \in[0, \delta]$, it follows that there exists $a_{3}$ such that for $a \in\left[0, a_{3}\right) f_{a}(t) t<h_{a_{1}} k_{a_{1}}^{2} /|\Omega|$ for $t \in(-\infty, \delta]$. Then if $\left\|u_{a}\right\|_{\infty}<\delta$ and $a \in\left[0, a_{3}\right)$ we are lead to the contradiction

$$
h_{a_{1}} k_{a_{1}}^{2} \leq M\left(\left\|u_{a}\right\|^{2}\right) \int_{\Omega}\left|\nabla u_{a}\right|^{2} d x=\int_{\Omega} f_{a}\left(u_{a}\right) u_{a} d x<h_{a_{1}} k_{a_{1}}^{2}
$$

and then the claim is proved.

\section{Proof of the main theorems}

In order to conclude the proof of Theorem 1.1 and 1.2, we need to show that the solution $u_{a}$ is positive for $a \in\left(0, a_{3}\right)$, decreasing $a_{3}$ if necessary. Indeed, let $\left\{a_{j}\right\} \subset\left(0, a_{3}\right)$ be a sequence with $a_{j} \rightarrow 0$ as $j \rightarrow \infty$, and let $u_{j}$ be a solution of $\left(\mathrm{AP}_{a}\right)$ with $a=a_{j}$. Setting $f_{j}\left(u_{j}\right)=f_{a_{j}}\left(u_{j}\right)$, we have

$$
\begin{cases}-M\left(\left\|u_{j}\right\|^{2}\right) \Delta u_{j}=f_{j}\left(u_{j}\right) & \text { in } \Omega, \\ u_{j}=0 & \text { on } \partial \Omega .\end{cases}
$$

From Lemma 3.2 we know that $u_{j}$ is bounded in $C^{1, \beta}(\Omega)$ for some $\beta \in(0,1)$. By the compact inclusion $C^{1, \beta}(\Omega) \subseteq C^{1, \gamma}(\Omega)$ for $0<\gamma<\beta$, we obtain a subsequence (still denoted by $u_{j}$ ) and a function $u \in C^{1, \gamma}(\Omega)$ such that $u_{j} \rightarrow u$ in $C^{1, \gamma}(\Omega)$.

Now using corollary 3.1 we can estimate

$$
-\Delta u_{j}=\frac{f_{j}\left(u_{j}\right)}{M\left(\left\|u_{j}\right\|^{2}\right)} \geq \frac{-a_{j}}{h_{a_{3}}} .
$$

Let $v_{j}$ be the solution of the problem

$$
\begin{cases}-\Delta v_{j}=k_{j}:=\frac{-a_{j}}{h_{a_{3}}} & \text { in } \Omega, \\ u_{j}=0 & \text { on } \partial \Omega,\end{cases}
$$

so that then $-\Delta v_{j} \leq-\Delta u_{j}$ and by the comparison principle for the Laplacian we conclude that

$$
v_{j} \leq u_{j} \quad \text { in } \Omega \text {. }
$$

Since $v_{j} \nearrow 0$ uniformly, this implies that $u \geq 0$.

Now notice that

- $\nabla u_{j}(x) \rightarrow \nabla u(x)$ uniformly in $\bar{\Omega}$,

- $\left\|u_{j}\right\| \rightarrow\|u\|$ and then $M\left(\left\|u_{j}\right\|^{2}\right) \rightarrow M\left(\|u\|^{2}\right)$,

- $\left\{f_{j}\left(u_{j}\right)\right\}$ is bounded in $L^{s}(\Omega), s>1$,

- $f_{j}\left(u_{j}\right) \rightarrow z$ in $L^{s}(\Omega)$,

- $f_{j}\left(u_{j}(x)\right) \rightarrow f_{0}(u(x))$ a.e. $x \in \Omega$,

where $f_{0}(t)=f(t)$ if $t \geq 0$, and $f_{0}(t)=0$ if $t<0$.

Having this in mind, we deduce that $z=f_{0}(u) \geq 0$, and for any $\varphi \in C_{0}^{\infty}(\Omega)$

$$
\begin{aligned}
M\left(\|u\|^{2}\right) \int_{\Omega} \nabla u \nabla \varphi d x & =\lim _{j \rightarrow+\infty} M\left(\left\|u_{j}\right\|^{2}\right) \int_{\Omega} \nabla u_{j} \nabla \varphi d x \\
& =\lim _{j \rightarrow+\infty} \int_{\Omega} f_{j}\left(u_{j}\right) \varphi d x=\int_{\Omega} z \varphi d x .
\end{aligned}
$$


As $\left\|u_{j}\right\|_{\infty} \geq \delta$ for all $j \in \mathbf{N}$, by Lemma 3.3, we derive that $\|u\|_{\infty} \geq \delta$, and so $u \not \equiv 0$, consequently $M\left(\|u\|^{2}\right)>0$, and from (4.1) we get

$$
\begin{cases}-\Delta u=\frac{z}{M\left(\|u\|^{2}\right)} & \text { in } \Omega, \\ u \geq 0 & \text { in } \Omega, \\ u=0 & \text { on } \partial \Omega .\end{cases}
$$

As $z \geq 0$, we obtain

$$
\begin{cases}-\Delta u \geq 0 & \text { in } \Omega, \\ u \geq 0 & \text { in } \Omega, \\ u=0 & \text { on } \partial \Omega .\end{cases}
$$

Now by the strong comparison principle for the Laplacian,

$$
u>0 \text { in } \Omega \text { and } \frac{\partial u}{\partial \eta}<0 \text { on } \partial \Omega,
$$

where $\partial / \partial \eta$ denotes the exterior normal derivative. This information together with the limit

$$
u_{j} \rightarrow u \text { in } C^{1, \tau}(\bar{\Omega})
$$

leads to $u_{j}(x)>0, x \in \Omega$, for $j$ large enough. Decreasing $a_{3}$ if necessary, the above analysis guarantees that $u_{a}$ is positive for $a \in\left(0, a_{3}\right)$. This completes the proof of the main Theorems.

\section{References}

[1] Ali, I., A. Castro, and R. Shivaji: Uniqueness and stability of nonnegative solutions for semipositone problems in a ball. - Proc. Amer. Math. Soc. 117:3, 1993, 775-782.

[2] Alves, C. O., F. J. S. A. CorrêA, and T. F. MA: Positive solutions for a quasilinear elliptic equation of Kirchhoff type. - Comput. Math. Appl. 49:1, 2005, 85-93.

[3] Alves, C. O., A. R. F. De Holanda, and J. A. Jefferson: Existence of positive solutions for a class of semipositone quasilinear problems through Orlicz-Sobolev space. - Proc. Amer. Math. Soc. 147:1, 2019, 285-299.

[4] Ambrosetti, A., and D. Arcoya: Remarks on non homogeneous elliptic Kirchhoff equations. - NoDEA Nonlinear Differential Equations Appl. 23:6, 2016, Art. 57, 11.

[5] Ambrosetti, A., and D. Arcoya: Positive solutions of elliptic Kirchhoff equations. - Adv. Nonlinear Stud. 17:1, 2017, 3-15.

[6] Ambrosetti, A., D. Arcoya, and B. Buffoni: Positive solutions for some semi-positone problems via bifurcation theory. - Differential Integral Equations 7:3-4, 1994, 655-663.

[7] Ambrosetti, A., and P. H. Rabinowitz: Dual variational methods in critical point theory and applications. - J. Funct. Anal. 14, 1973, 349-381.

[8] Anello, G.: On a perturbed Dirichlet problem for a nonlocal differential equation of Kirchhoff type. - Bound. Value Probl. 2011, 2011, Art. ID 891430, 10.

[9] Anuradha, V., D. D. Hai, and R. Shivaji: Existence results for superlinear semipositone BVPs. - Proc. Amer. Math. Soc. 124:3, 1996, 757-763.

[10] Caldwell, S., A. Castro, R. Shivaji, and S. Unsurangsie: Positive solutions for classes of multiparameter elliptic semipositone problems. - Electron. J. Differential Equations 2007, 2007, No. 96, 10.

[11] Castro, A., D. G. De Figueiredo, and E. Lopera: Existence of positive solutions for a semipositone p-Laplacian problem. - Proc. Roy. Soc. Edinburgh Sect. A 146:3, 2016, 475-482.

[12] Castro, A., and R. ShivaJi: Nonnegative solutions for a class of nonpositone problems. Proc. Roy. Edinburgh Sect. A 108:3-4, 1988, 291-302. 
[13] Cheng, B., X. Wu, and J. LiU: Multiple solutions for a class of Kirchhoff type problems with concave nonlinearity. - NoDEA Nonlinear Differential Equations Appl. 19:5, 2012, 521-537.

[14] Chu, K. D., D. D. Hai, and R. Shivaji: Uniqueness of positive radial solutions for infinite semipositone $p$-Laplacian problems in exterior domains. - J. Math. Anal. Appl. 472, 2019, $510-525$.

[15] Colasuonno, F., and P. PuCCI: Multiplicity of solutions for $p(x)$-polyharmonic elliptic Kirchhoff equations. - Nonlinear Anal. 74:17, 2011, 5962-5974.

[16] CorrêA, F. J. S. A., and G. M. Figueiredo: On an elliptic equation of $p$-Kirchhoff type via variational methods. - Bull. Austral. Math. Soc. 74:2, 2006, 263-277.

[17] Costa, D. G., H. R. Quorin, and H. Tehrani: A variational approach to superlinear semipositone elliptic problems. - Proc. Amer. Math. Soc. 145:6, 2017, 2661-2675.

[18] DAI, G., and R. HAO: Existence of solutions for a $p(x)$-Kirchhoff-type equation. - J. Math. Anal. Appl. 359:1, 2009, 275-284.

[19] De Figueiredo, D. G., J.-P. Gossez, and P. Ubilla: Local "superlinearity" and "sublinearity" for the $p$-Laplacian. - J. Funct. Anal. 257:3, 2009, 721-752.

[20] Garcia Azorero, J., and I. Peral Alonso: Some results about the existence of a second positive solution in a quasilinear critical problem. - Indiana Univ. Math. J. 43:3, 1994, 941-957.

[21] Iturriaga, L., and E. Massa: On necessary conditions for the comparison principle and the sub- and supersolution method for the stationary Kirchhoff equation. - J. Math. Phys. 59:1, 2018, 011506, 6 .

[22] IturRiaga, L., and E. Massa: Sobolev versus Hölder local minimizers in degenerate Kirchhoff type problems. - J. Differential Equations 269:5, 2020, 4381-4405.

[23] Kajikiya, R., and E. Ko: Existence of positive radial solutions for a semipositone elliptic equation. - J. Math. Anal. Appl. 484, 2020, 123735.

[24] Kirchioff, G.: Mechanik. - Teubner, Leipzig, 1883.

[25] Ladyzhenskaya, O. A., and N. N. Ural'tseva: Linear and quasilinear elliptic equations. Academic Press, New York-London, 1968.

[26] Lieberman, G. M.: Boundary regularity for solutions of degenerate elliptic equations. - Nonlinear Anal. 12:11, 1988, 1203-1219.

[27] MA, T.F.: Remarks on an elliptic equation of Kirchhoff type. - Nonlinear Anal. 63, 2005, e1967-e1977.

[28] Madeira, G., and A. Nunes: Concave-convex structure for degenerate, singular or discontinuous elliptic Kirchhoff equations. - Preprint, 2019.

[29] Perera, K., R. Shivaji, and I. Sim: A class of semipositone $p$-Laplacian problems with a critical growth reaction term. - Adv. Nonlinear Anal. 9, 2020, 516-525.

[30] Santos Júnior, J.R., and G. Siciliano: Positive solutions for a Kirchhoff problem with vanishing nonlocal term. - J. Differential Equations 265:5, 2018, 2034-2043.

[31] Song, S.-Z., S.-J. Chen, and C.-L. TANG Existence of solutions for Kirchhoff type problems with resonance at higher eigenvalues. - Discrete Contin. Dyn. Syst. 36:11, 2016, 6453-6473.

[32] TANG, X. H., and B. Cheng: Ground state sign-changing solutions for Kirchhoff type problems in bounded domains. - J. Differential Equations 261:4, 2016, 2384-2402.

[33] Villaggio, P.: Mathematical models for elastic structures. - Cambridge Univ. Press, Cambridge, 1997. 